\title{
Deshidratación de quinua (Chenopodium quinoa Willd) asistido por infrarrojo
}

Quinoa dehydration (Chenopodium quinoa Willd) assisted by infrared

\author{
Juan Federico Ramos Gómez ${ }^{1}$, Ángel Oscar Peña Rivera ${ }^{1}$
}

\section{RESUMEN}

Objetivo: Determinar el efecto de la temperatura de aire y potencia de rayos infrarrojos sobre el secado de la quinua variedad Hualhuas. Material y Métodos: Para la conducción del experimento se utilizó el diseño completamente aleatorizado con arreglo factorial de $3 \times 3$, luego de haber preparado las muestras a través del remojado, desaponificación por lavado y enjuagado. Los factores de prueba fueron la temperatura del aire de secado, con tres niveles $40^{\circ} \mathrm{C}, 50^{\circ} \mathrm{C}$ y $60^{\circ} \mathrm{C}$, y la potencia de los rayos infrarrojos 0,250 y $500 \mathrm{~W}$. Las variables respuesta consideradas fueron el tiempo de secado y la humedad de equilibrio final de los granos de quinua. Resultados: El tiempo de secado al utilizar $40^{\circ} \mathrm{C}$ de temperatura sin radiación infrarroja fue de $6,75 \mathrm{~h}, 5,0 \mathrm{~h}$ con $50^{\circ} \mathrm{C}$ y $3,75 \mathrm{~h}$ con $60^{\circ} \mathrm{C}$. Para $250 \mathrm{~W}$ de radiación infrarroja con $40^{\circ} \mathrm{C}$ el tiempo de secado fue de $3,50 \mathrm{~h}$ y $2,50 \mathrm{~h}$ con $60^{\circ} \mathrm{C}$. Para $500 \mathrm{~W}$ con $40^{\circ} \mathrm{C} 2,0 \mathrm{~h}$ y para $60^{\circ} \mathrm{C} 1,50 \mathrm{~h}$. Las humedades finales alcanzadas para todos los casos son diferentes. Conclusiones: Al incrementar la temperatura del aire de secado y de la potencia de la radiación de los rayos infrarrojos en forma simultánea, se logra disminuir el tiempo de secado, hasta en un $77,7 \%$ comparado con secado solo con aire caliente a $40{ }^{\circ} \mathrm{C}$ y alcanzar humedades de equilibrio tan bajas como $0,05 \mathrm{~kg} \mathrm{H}_{2} \mathrm{O} / \mathrm{kg} \mathrm{m}$.s. El menor tiempo de secado de 1,50 $\mathrm{h}$; fue obtenido utilizado $500 \mathrm{~W}$ de potencia con $60^{\circ} \mathrm{C}$.

Palabras clave: Quinua, quinua secado, secado infrarrojo, quinua infrarrojo

\section{ABSTRACT}

Objective: To determine the effect of air temperature and infrared ray potency on the drying of the Hualhuas quinoa variety. Material and Methods: For the conduct of the experiment, the completely randomized design with a $3 \times 3$ factorial arrangement was used, after having prepared the samples through soaking, deponification by washing and rinse. The test factors were the temperature of the drying air, with three levels $40^{\circ} \mathrm{C}, 50^{\circ} \mathrm{C}$ and $60^{\circ} \mathrm{C}$, and the power of the infrared rays $0 ; 250$ and $500 \mathrm{~W}$. The response variables considered were the drying time and the humidity of final balance of quinoa grains. Results: The drying time when using $40^{\circ} \mathrm{C}$ temperature without infrared radiation was $6,75 \mathrm{~h} ; 5,0 \mathrm{~h}$ with $50^{\circ} \mathrm{C}$ and $3,75 \mathrm{~h}$ with $60^{\circ} \mathrm{C}$. For $250 \mathrm{~W}$ of infrared radiation with $40^{\circ} \mathrm{C}$ the drying time was from $3,50 \mathrm{~h}$ and $2,50 \mathrm{~h}$ with $60^{\circ} \mathrm{C}$. For $500 \mathrm{~W}$ with $40^{\circ} \mathrm{C} 2,0 \mathrm{~h}$ and for $60^{\circ} \mathrm{C} 1,50 \mathrm{~h}$. The final humidity reached for all cases is different. Conclusions: By increasing the temperature of the drying air and the radiation power of the infrared rays simultaneously, it is possible to reduce the drying time, up to $77,7 \%$ compared to drying only with hot air at $40^{\circ} \mathrm{C}$ and reach equilibrium humidities as low as $0,05 \mathrm{~kg} \mathrm{H} \mathrm{O}_{2} \mathrm{~kg}$ m.s. The shortest drying time of 1,50 h; $500 \mathrm{~W}$ of power was obtained with $60^{\circ} \mathrm{C}$.

Keywords: Quinoa, Quinoa drying, infrared drying, infrared quinoa. 


\section{INTRODUCCIÓN}

La quinua (Chenopodium quinoa W.) es una planta alimenticia, nativa de los Andes americanos; su valor nutritivo radica, en la calidad de la proteína y posee en su combinación una mayor proporción de aminoácidos (lisina, arginina, histidina y cistina) esenciales para la alimentación humana (Manco, 1988), se cultiva principalmente para la producción de grano que se consume como sustituto del arroz, o como harina, es conocida como grano de los incas, porque se constituyó en la principal fuente alimenticia del reino incaico (Mujica, 1993).

La variedad de quinua Hualhuas, fue registrada con la clave UNC-H20 B-74, tiene su origen en el UNC$\mathrm{H}-20-\mathrm{P}-69$, obtenida por selección de sus segregantes en el año 1974. A partir de 1975 Florencio Herquinio realizo una rigurosa selección masal y genealógica por sus características agronómica ideales para el agricultor que se dedica a cultivar y en honor al suelo natal que lo vio nacer bautiza al fruto de su sabiduría con el nombre de Hulahuas (INIA, 2006).

El aspecto del grano es opaco, con perigonio y percarpio crema, episperma y perisperma blanco, forma del borde afilado con forma cilíndrica, diámetro medio del grano de $2,10 \mathrm{~mm}$, rendimiento de semillas por planta de 52,80 g (Apaza, 2013). Quiroga, Escalera y Arteaga (2010) demostraron que el episperma recubre al grano de quínua. En los granos sin procesar el espesor del episperma varía de $20 \mu \mathrm{m}$ en la parte central de las caras hasta más de $100 \mu \mathrm{m}$ en los extremos, cerca al embrión. La capa más externa del episperma es la de mayor espesor. Esta capa puede fragmentarse durante el proceso de manipulación de la materia prima, su remoción es efectiva solo a través de procesos físicos y químicos.

El proceso tradicional de desaponificación, es por vía húmeda, que demanda $5-14 \mathrm{~m}^{3}$ de agua por TM de quinua procesada, y genera volúmenes considerables de efluentes contaminados con saponina (Quiroga, Escalera y Arteaga, 2010), consiste en lavados sucesivos del grano con agua, hasta que la espuma desaparezca, lo que rehidrata los granos en promedio hasta el $30 \%$ de humedad. A nivel industrial se realiza con agitación mecánica y turbulencia, seguido de secado, que demanda de periodos de tiempo prolongados y costos elevados por consumo de energía (Pereira, 2011).

En el secado, la temperatura $(T)$ y la humedad relativa (HR) del aire son vitales, generalmente conforme se incrementa la $T$, se reduce la HR y se acelera la eliminación de humedad, la velocidad de aire dentro del secador es un factor trascendental por la función de transmisor de energía y transportador de humedad saliente del material, la velocidad apropiada se encuentra entre 2 a $3 \mathrm{~m} / \mathrm{s}$.
(Perry, 1984), mejorar sus capacidades de expresión y comprensión de textos, desarrollar la imaginación, fantasía e historias reales de los niños, y replicar estrategias didácticas contenidas en el programa experimental "escritura creativa", en otras instituciones educativas del Perú, generando con ello un efecto multiplicador Espinoza (2011), secó murtilla haciendo uso de aire caliente asistido por infrarrojo, habiendo obtenido los tiempos de secado que se muestran en el Tabla 1. Pitchaporn, Siriamornpun y Meeso (2011), secaron hojas de té, sometiéndolo a dos métodos de secado diferentes, aire caliente y FIR-HA. Para cada método de secado, usaron $100 \mathrm{~g}$ de hojas frescas. En el secado FIR-HA, las hojas se secaron en un secador combinado aire caliente y FIR-HA a una intensidad FIR de $5 \mathrm{~kW} / \mathrm{m}^{2}$ (energía FIR irradiada por área de superficie FIR del calentador) temperatura HA de $40^{\circ} \mathrm{C}$, velocidades de HA de $1,5 \mathrm{~m} / \mathrm{s}$, y tiempo de secado de $60 \mathrm{~min}$. Después del secado, estas muestras (denominadas "té de morera") se dejaron enfriar a temperatura ambiente antes de la extracción para la determinación de compuestos bioactivos. Estos resultados son favorables y pueden ser utilizados también en el secado de quinua.

Sriariyakul, Swasdisevi, Devahastin y Soponronnarit (2016), secaron mucílago de aloe vera con aire caliente con y sin la aplicación de radiación infrarroja lejana $(F I R)$ y campo eléctrico de alta tensión (HVEF). El puré se secó a varias velocidades de aire $(0,2 ; 0,6$ y $1 \mathrm{~m} / \mathrm{s})$, y temperaturas superficiales controladas $(50 ; 60$ y 70 ${ }^{\circ} \mathrm{C}$ ), así como intensidades de campo eléctrico de alta tensión $(0 ; 1,88$ y 3,75 kV/cm). Se realizó una evaluación comparativa de la cinética de secado, el consumo específico de energía (SEC) y la calidad (en términos de color, aloína, polisacáridos totales como recuentos microbianos) de los productos sometidos a diferentes procesos de secado. La intensidad del campo eléctrico tuvo un efecto significativo sobre la cinética de secado, la SEC y la calidad del producto seco. Por otro lado, HVEF exhibió un efecto dominante en la cinética de secado solo a una velocidad de aire menor $(0,2$ $\mathrm{m} / \mathrm{s}$ ).

La aplicación de HVEF podría reducir significativamente la SEC del proceso de secado a una velocidad del aire de 0,2 m/s. La condición óptima para el secado del puré de aloe vera con aire caliente en combinación con FIR y HVEF fue el uso de una velocidad de aire de $0,2 \mathrm{~m} / \mathrm{s}$, temperatura de $70^{\circ} \mathrm{C}$ y una intensidad de campo eléctrico de alta tensión de $3,75 \mathrm{kV} / \mathrm{cm}$.

Durante la desaponificación, la quinua alcanza entre $29 \%$ a $34 \%$ de humedad; y debe ser secado a niveles menores al 13\% (Soria, Marcial y Peñaloza, 1990), para evitar ataque de microorganismos, 
fermentaciones y germinaciones que deterioren la calidad; esta etapa que se hace por exposición al sol toma en promedio cuatro días (Romo, Rosero et al, 2006).

En secadores técnicos la calefacción puede provenir de un intercambiador de calor que operan con gas licuado, o de una resistencia eléctrica con un termostato adecuado, que luego es circulado al interior del secador (Salas, 2011). Cerrón (2013), determinó que al secar quinua con aire a temperatura ambiente se requiere en promedio de $7,25 \mathrm{~h}$ (temperatura media de aire 17 ${ }^{\circ} \mathrm{C} \pm 2{ }^{\circ} \mathrm{C}$ ). Con aire a $30^{\circ} \mathrm{C}$ requirió de 5,75 a 6,0 h., con aire a $50^{\circ} \mathrm{C}$ se redujo a 4,00 h. Miranda, Gálvez, López, Parada, Sanders, Aranda, Uribe y Di Scala (2010), secaron quinua a diferentes temperaturas, los resultados alcanzados fueron que la operación de secado conduce a reducciones de $10 \%$ en proteínas, $12 \%$ en grasa y $27 \%$ en fibras y cenizas. La pérdida de proteína podría deberse a desnaturalización o cambios en la solubilidad durante el secado, o a la liberación de aminoácidos de las proteínas después de la desnaturalización que podría reaccionar con otros compuestos químicos, como los azúcares, para producir melanoidinas a través de la reacción de Maillard.

\section{MATERIALY MÉTODOS}

Para el desamargado de quinua, se procedió con un remojado previo de los granos por tiempo de 15 minutos utilizando una proporción de 3 partes de agua y una de quinua, a temperatura ambiente, acorde a lo sugerido por Borda y Gamarra (2002).

Luego los granos fueron desaponificados, en un equipo que utiliza el principio de agitación por bombeo de agua con caudal controlado. El lavado se realizó con 3 partes de agua y uno de quinua
(3/1), por el tiempo de 15 minutos utilizando un caudal de bombeo de agua de $30 \mathrm{~L} / \mathrm{min}$. Seguido de un enjuague, con la misma proporción de agua/quinua, y los mismos parámetros de tiempo y caudal de agua.

Finalizado el lavado y enjuagado, se verificó el contenido de saponina de los granos lavados, haciendo uso del método rápido. En todos los casos la quinua lavada se encontró libre de saponina.

La quinua desamargada, luego se cargó en bandejas de secado y se llevó a un secador equipado con luminarias de rayos infrarrojos, y flujo de aire caliente controlables, donde se procedió con la deshidratación. Para la conducción del experimento de secado se utilizó un diseño completamente aleatorizado con arreglo factorial de $3 \times 3$. Los factores de prueba fueron temperatura del aire de secado $\left(40^{\circ} \mathrm{C} ; 50\right.$ ${ }^{\circ} \mathrm{C}$ y $60^{\circ} \mathrm{C}$ ) y Potencia de rayos infrarrojos incidentes $(0 ; 250$ y $500 \mathrm{~W})$ que resulto en las siguientes combinaciones: $40^{\circ} \mathrm{C} \times 0 \mathrm{~W}, 40^{\circ} \mathrm{C} \times$ $400 \mathrm{~W}, 40^{\circ} \mathrm{C} \times 800 \mathrm{~W}, 50^{\circ} \mathrm{C} \times 0 \mathrm{~W}, 50^{\circ} \mathrm{C} \times 400 \mathrm{~W}, 50$ ${ }^{\circ} \mathrm{C}$ x $800 \mathrm{~W}$ y $60^{\circ} \mathrm{C} \times 0 \mathrm{~W}, 60^{\circ} \mathrm{C} \times 400 \mathrm{~W}, 60^{\circ} \mathrm{C} \mathrm{x}$ $800 \mathrm{~W}$.

Se utilizó balanza analítica sartorius con centésima de sensibilidad, secador de cabina con temperatura y velocidad de aire regulables, termómetros tipo termistor, cronómetro, pipetas de 5,0 y $10 \mathrm{~mL}$, tubos de prueba de $16 \times 160 \mathrm{~mm}$ provistos de tapón.

\section{RESULTADOS}

Los resultados de las curvas de secado de quinua a temperaturas de $40^{\circ} \mathrm{C} ; 50^{\circ} \mathrm{C}$ y $60^{\circ} \mathrm{C}$ con 0,250 y $500 \mathrm{~W}$ de radiación infrarroja, se muestran en la Figura $1 \mathrm{~A}, \mathrm{~B}$ y $\mathrm{C}$.

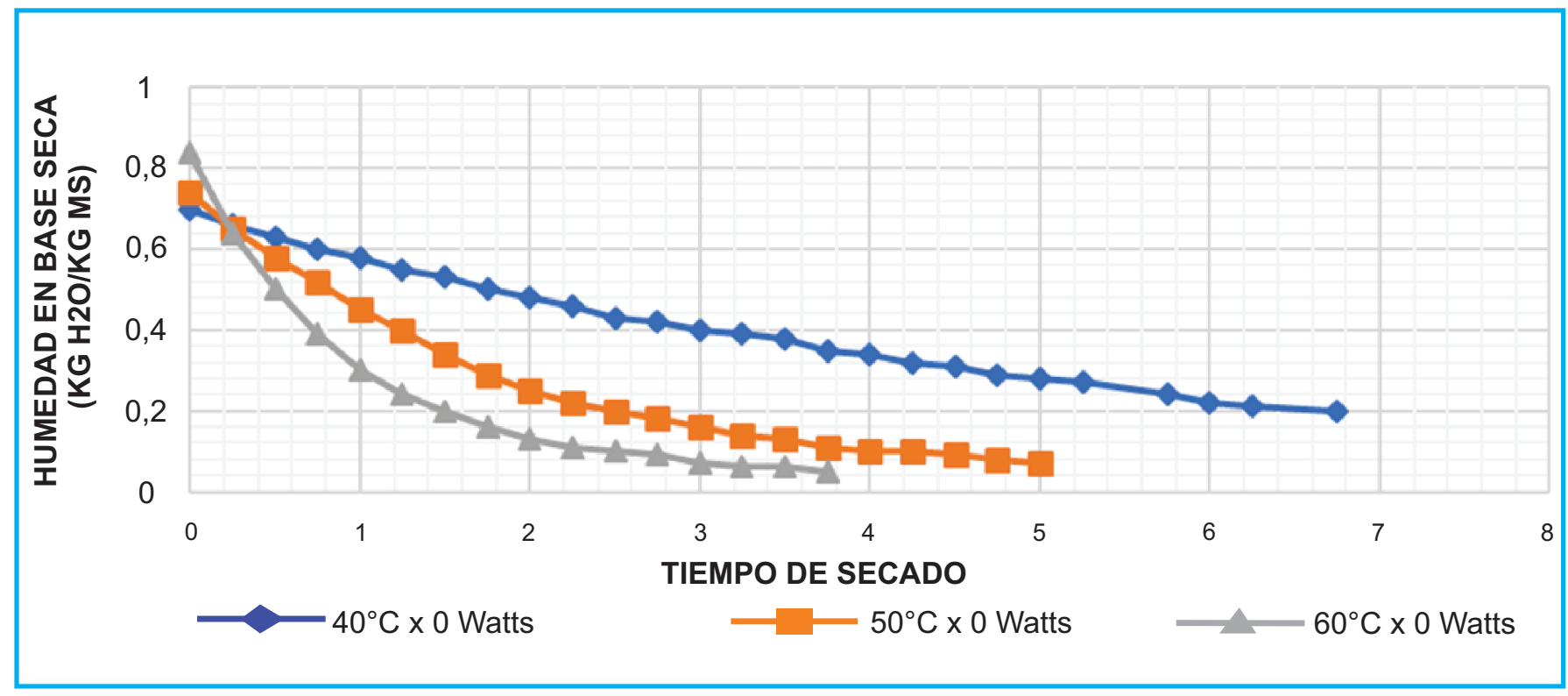



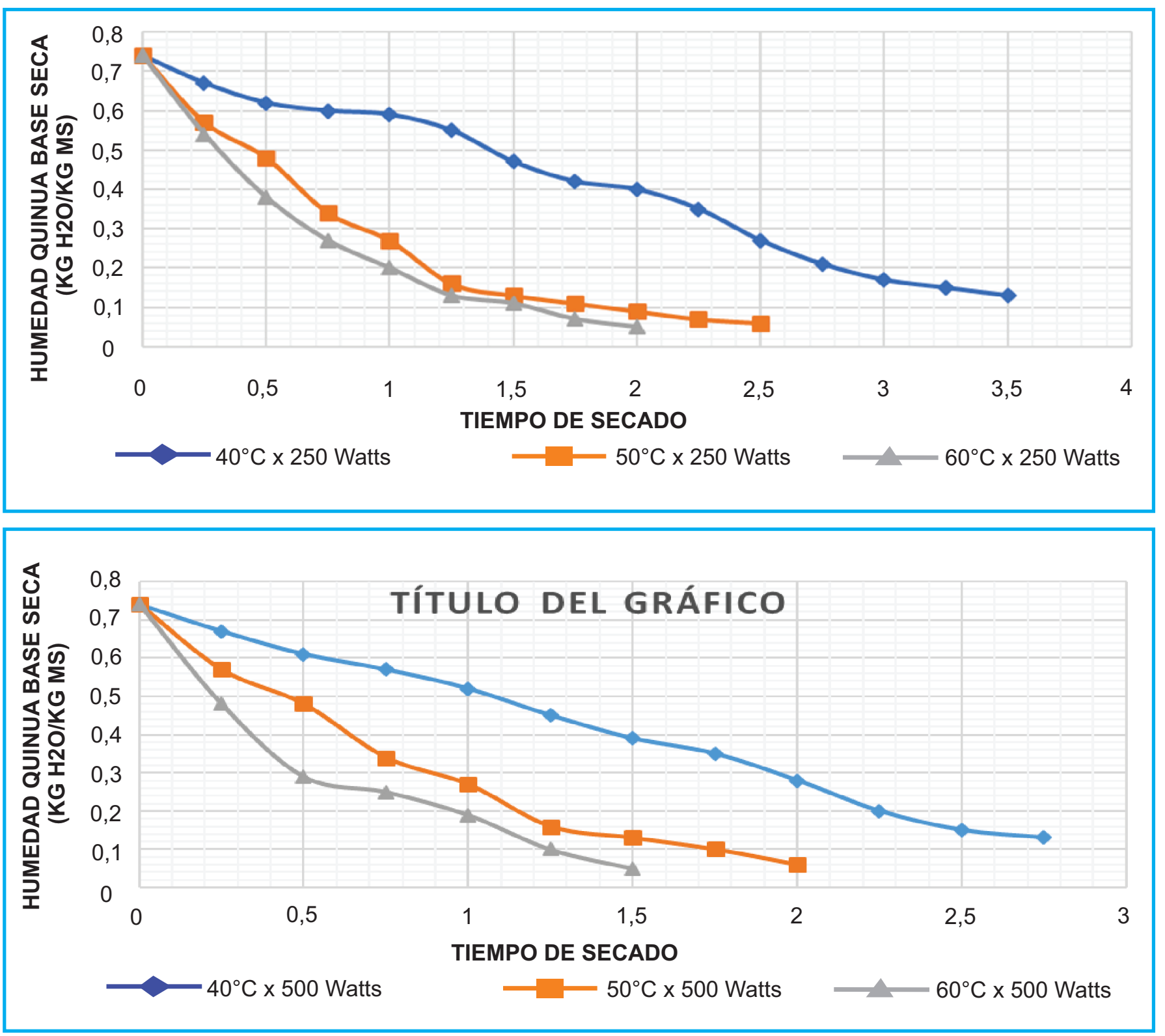

Figura 1. Curvas de secado de quinua a temperaturas de $40^{\circ} \mathrm{C} ; 50^{\circ} \mathrm{C}$ y $60^{\circ} \mathrm{C}$, con $0 \mathrm{~W}(\mathrm{~A}), 250 \mathrm{~W}$ (B) y $500 \mathrm{~W}(\mathrm{C})$

Un resumen del tiempo de secado medio y la humedad de equilibrio alcanzado, en función de la totalidad de tratamientos utilizados en el experimento es mostrado en la Tabla 1 , mientras los tiempos de secado determinados como variables respuesta para cada tratamiento se muestra en la Tabla 2.

Tabla 1. Tiempos de secado y humedades de equilibrio promedio en deshidratación de quinua a temperaturas y radiaciones infrarrojas diferentes

\begin{tabular}{lcc}
$\begin{array}{c}\text { Parámetro de } \\
\text { secado }\end{array}$ & $\begin{array}{c}\text { Tiempo de } \\
\text { secado (h) }\end{array}$ & $\begin{array}{c}\text { Humedad de equilibrio } \\
\text { (kg. H20/kg m.s.) }\end{array}$ \\
\hline $40{ }^{\circ} \mathrm{C} \times 0 \mathrm{~W}$ & 6,5 & 0,20 \\
$50{ }^{\circ} \mathrm{C} \times 0 \mathrm{~W}$ & 5,00 & 0,07 \\
$60{ }^{\circ} \mathrm{C} \times 0 \mathrm{~W}$ & 3,75 & 0,05 \\
$40{ }^{\circ} \mathrm{C} \times 250 \mathrm{~W}$ & 3,50 & 0,13 \\
$50{ }^{\circ} \mathrm{C} \times 250 \mathrm{~W}$ & 2,50 & 0,06 \\
$60{ }^{\circ} \mathrm{C} \times 250 \mathrm{~W}$ & 2,00 & 0,05 \\
$40{ }^{\circ} \mathrm{C} \times 500 \mathrm{~W}$ & 3,00 & 0,12 \\
$50{ }^{\circ} \mathrm{C} \times 500 \mathrm{~W}$ & 2,00 & 0,06 \\
$60{ }^{\circ} \mathrm{C} \times 500 \mathrm{~W}$ & 1,50 & 0,05 \\
\hline
\end{tabular}


Tabla 2. tiempos de secado determinados como variables respuesta para cada tratamiento se muestra en la Tabla 2.

\begin{tabular}{cccc} 
Radiación & \multicolumn{3}{c}{ Temperatura de secado } \\
\cline { 2 - 4 } infrarroja & $\mathbf{4 0}{ }^{\circ} \mathbf{C}$ & $\mathbf{5 0}{ }^{\circ} \mathbf{C}$ & $\mathbf{6 0}{ }^{\circ} \mathbf{C}$ \\
\hline \multirow{2}{*}{$\mathbf{~ W ~ W ~}$} & 6,77 & 5,15 & 3,66 \\
& 6,72 & 4,86 & 3,85 \\
$\mathbf{2 5 0} \mathbf{~ W}$ & 3,57 & 2,59 & 2,10 \\
& 3,42 & 2,42 & 1,92 \\
& 3,31 & 2,12 & 1,60 \\
$\mathbf{5 0 0} \mathbf{~ W}$ & 2,72 & 1,90 & 1,41 \\
\hline
\end{tabular}

El análisis de varianza mostró diferencias significativas entre los diferentes niveles de los factores principales y para los diversos tratamientos de la interacción (AB). La prueba de comparación múltiple de medias del tiempo de secado causado por los diversos niveles de los factores radiación infrarroja y temperatura del aire de secado, se muestra en la Tabla 3.

Tabla 3. Efecto de la radiación infrarroja y la temperatura de secado en el tiempo de secado

\begin{tabular}{lccc} 
Parámetros & $\begin{array}{c}\text { Niveles } \\
\text { de factor }\end{array}$ & N & Promedio \\
\hline $\begin{array}{l}\text { Radiación } \\
\text { infrarroja }\end{array}$ & $500 \mathrm{~W}$ & 6 & $2,167^{\mathrm{a}}$ \\
\hline & $0 \mathrm{~W}$ & 6 & $5,167^{\circ}$ \\
\hline $\begin{array}{l}\text { Temperatura } \\
\text { aire de } \\
\text { secado }\end{array}$ & 3,42 & 6 & $2,417^{\mathrm{d}}$ \\
\hline
\end{tabular}

Para conocer el efecto de cada uno de los tratamientos (combinación de los dos factores principales y sus diferentes niveles de factor), en el tiempo de secado, se aplicó una PCMM de Duncan, cuyo resultado es mostrado en la tabla 4.
Tabla 4. Efecto de los tratamientos en el tiempo de secado

\begin{tabular}{lccc}
$\begin{array}{c}\text { Radiación } \\
\text { infrarroja }\end{array}$ & $\begin{array}{c}\text { Temperatura de } \\
\text { aire de secado }\end{array}$ & $\mathrm{N}$ & Promedio \\
\hline $500 \mathrm{~W}$ & $60{ }^{\circ} \mathrm{C}$ & 2 & $1,5^{\mathrm{a}}$ \\
\hline $250 \mathrm{~W}$ & $60{ }^{\circ} \mathrm{C}$ & 2 & $2,0^{\mathrm{b}}$ \\
$500 \mathrm{~W}$ & $50{ }^{\circ} \mathrm{C}$ & 2 & $2,0^{\mathrm{b}}$ \\
$250 \mathrm{~W}$ & $50{ }^{\circ} \mathrm{C}$ & 2 & $2,5^{\mathrm{c}}$ \\
$500 \mathrm{~W}$ & $40{ }^{\circ} \mathrm{C}$ & 2 & $3,0^{\mathrm{d}}$ \\
$250 \mathrm{~W}$ & $40{ }^{\circ} \mathrm{C}$ & 2 & $3,5^{\mathrm{e}}$ \\
$0 \mathrm{~W}$ & $60{ }^{\circ} \mathrm{C}$ & 2 & $3,75^{\circ}$ \\
$0 \mathrm{~W}$ & $50{ }^{\circ} \mathrm{C}$ & 2 & $5,0^{\mathrm{f}}$ \\
$0 \mathrm{~W}$ & $40^{\circ} \mathrm{C}$ & 2 & $6,75^{9}$ \\
\hline
\end{tabular}

\section{DISCUSIÓN}

La Figura $1 \mathrm{~A}$, B y $\mathrm{C}$ muestran en general que a mayor temperatura de aire, el tiempo de secado obtenido tiende a ser menor, tanto con 0; 250 o $500 \mathrm{~W}$ de radiación infrarroja.

Los tiempos de secado obtenidos para la quinua, cuando fueron deshidratados sin radiación infrarroja $(0 \mathrm{~W})$, fueron de 6,75 horas (aire a 40 ${ }^{\circ} \mathrm{C}$ ), habiéndose reducido a $5,0 \mathrm{~h}$ para $50{ }^{\circ} \mathrm{C}$, y hasta 3,75 horas para $60{ }^{\circ} \mathrm{C}$. al incorporar radiación infrarroja de $250 \mathrm{~W}$, los tiempos de secado se reducen a $3,50 \mathrm{~h}$ y $2,0 \mathrm{~h}$ incluso. Esta tendencia continúa para cuando se aplica $500 \mathrm{~W}$, alanzando finalmente un tiempo medio de 1,50 h para el tratamiento de $60{ }^{\circ} \mathrm{C} \times 500 \mathrm{~W}$. Aquí podemos deducir que existe un efecto inverso de la combinación de temperatura del aire y la potencia de radiación infrarroja en el tiempo de secado hasta alcanzar la humedad de equilibrio correspondiente.

Estos resultados experimentales alcanzados son cercanos a los obtenidos por Cerrón (2013), quien con aire a temperatura ambiente requirió en promedio de 7,25 h (temperatura media de aire 17 ${ }^{\circ} \mathrm{C} \pm 2{ }^{\circ} \mathrm{C}$ ), con aire a $30^{\circ} \mathrm{C}$ secó en 5,75 a $6,0 \mathrm{~h}$., con aire a $50^{\circ} \mathrm{C}$, el tiempo de secado fue de $4,0 \mathrm{~h}$. En este caso, la explicación de un menor tiempo de secado para alcanzar la humedad de equilibrio, cuando la quinua es secada con aire más caliente, se encuentra en la teoría propuesta por Perry (1984), quien manifiesta que cuando se calienta más el aire, este alcanza una humedad relativa más baja, lo que hace que se incremente la capacidad de absorción de humedad por parte del 
aire y se confirma con lo sostenido por Geankoplis (2002), que indica que cuando se calienta el aire la presión parcial del vapor de agua en el aire, tiende a ser menor comparado con la presión del vapor de agua a la misma temperatura, siendo que esta última (la presión del vapor de agua a la misma temperatura), es la que prevalece en la superficie de la quinua que es secada (por lo menos durante el periodo de secado a velocidad constante), hace que las moléculas de agua se transfieran rápidamente hacia el seno del aire.

En este caso la velocidad del aire se ha mantenido constante en $2,0 \mathrm{~m} / \mathrm{s}$, para todos los casos, entonces la función sostenida por Perry (1984) de ser transportador simultáneo de calor y materia, también se ha mantenido constante para todas las temperaturas de secado, luego la capa límite de secado que existe entre el material a secar y el aire de secado en todos los casos fue el mismo, no habiendo generado un espesor más delgado de la capa límite que habría favorecido en una más rápida remoción de la humedad. La velocidad de aire utilizada en el secador de bandejas se fijó dentro del rango sugerido por Perry (1984).

Cuando los granos de quinua fueron secados, a temperaturas de $40^{\circ} \mathrm{C} ; 50^{\circ} \mathrm{C}$ y $60^{\circ} \mathrm{C}$, con $250 \mathrm{~W}$ de radiación infrarroja, los tiempos se redujeron a 3,50 $\mathrm{h} ; 2,50 \mathrm{~h}$ y $2,0 \mathrm{~h}$, lo que nos permite afirmar que la reducción del tiempo de secado en función de $0 \mathrm{~W}$ de radiación infrarroja, fue del orden del $48 \%$ para la temperatura de $40{ }^{\circ} \mathrm{C}, 50 \%$ para el secado con aire a $50^{\circ} \mathrm{C}$ y de $47 \%$ para el secado con aire de $60^{\circ} \mathrm{C}$.

Al secar quinua con temperaturas de $40^{\circ} \mathrm{C} ; 50^{\circ} \mathrm{C}$ y $60{ }^{\circ} \mathrm{C}$, con $500 \mathrm{~W}$ de radiación infrarroja, los tiempos se redujeron a $3,0 \mathrm{~h} ; 2,0 \mathrm{~h}$ y $1,5 \mathrm{~h}$, que representan el $44 \%$ del obtenido con $40{ }^{\circ} \mathrm{C}$ y $0 \mathrm{~W}$, $40 \%$ para el secado con aire a $50{ }^{\circ} \mathrm{C}$ y $0 \mathrm{~W}$ y $40 \%$ para el secado con aire de $60{ }^{\circ} \mathrm{C}$ y $0 \mathrm{~W}$. Otra característica observada durante la investigación fue que los granos de quinua conservan un color crema sin la presencia de puntos negros que se presentó cuando fueron secadas solo con aire caliente.

La prueba de comparación múltiple de medias de Duncan al efecto generado por la radiación infrarroja en el tiempo de secado (Tabla 4), se deduce que el menor tiempo de secado se logra utilizando 500 Watts $(2,167 \mathrm{~h})$, seguido de la radiación de 250 Watts $(2,667 \mathrm{~h})$, finalmente con 0 Watts $(5,167 \mathrm{~h})$. El efecto generado por los diversos niveles de la temperatura de aire en el tiempo de secado, mostrado en la Tabla 4 indica que el menor tiempo de secado se logra utilizando $60^{\circ} \mathrm{C}(2,417$ h), seguido de $50^{\circ} \mathrm{C}(3,166 \mathrm{~h})$, finalmente con $40^{\circ} \mathrm{C}$ $(4,42 \mathrm{~h})$. Existiendo un correlato inverso entre estas dos variables, a mayor temperatura menor tiempo de secado.
La PCMM de Duncan del efecto de los tratamientos (combinación de los dos factores principales y sus diferentes niveles de factor), sobre el tiempo de secado muestra que el menor tiempo de secado $(1,5 \mathrm{~h})$ se logra combinando $500 \mathrm{~W}$ de radiación infrarroja con $60{ }^{\circ} \mathrm{C}$, diferente a los otros tratamientos. Los tratamientos que se acercan al primero con $2,0 \mathrm{~h}$ de secado, fue generado por 250 $\mathrm{W} \times 60{ }^{\circ} \mathrm{C}$ y con $500 \mathrm{~W}$ con $50{ }^{\circ} \mathrm{C}$, pero los resultados alcanzado son estadísticamente diferentes al primero.

La reducción del tiempo de secado al utilizar simultáneamente aire caliente y radiación infrarroja se debe fundamentalmente a que la segunda penetra al interior de los granos de quinua donde produce vibración y consiguientemente calentamiento del agua, que se difunde rápidamente a la superficie de la quinua, desde donde el aire más caliente tiene la oportunidad de aceptar una mayor cantidad de vapor de agua que el aire a menor temperatura. Esta combinación lograda reduce a su vez el tiempo de uso del secador, que en menor tiempo está disponible para recibir un nuevo lote de quinua que secar, dándose un aparente incremento en la capacidad de secado.

Los tiempos obtenidos como resultado, se encuentran muy cercanos al logrado por Espinoza (2011), quien secando murtilla obtuvo el tiempo de $90 \mathrm{~min}$ (que fue el menor), haciendo uso de $60^{\circ} \mathrm{C}$ de temperatura y $800 \mathrm{~W}$ de radiación infrarroja y 120 min utilizando $50{ }^{\circ} \mathrm{C} \times 800 \mathrm{~W}$. En general, la investigación demostró que el incremento de temperatura y de la potencia de radiación en forma simultánea genera una reducción en el tiempo de secado de quinua, tendencia evidenciada también por Espinoza, porque la asistencia de los rayos infrarrojos utilizado en el experimento tiene radiación electromagnética con longitud de onda de 50 a $1000 \mathrm{~nm}$ (infrarrojo lejano, más cercano al microondas), que ocasiona un fácil ingreso al interior de la quinua por la baja frecuencia que posee (menor al de la luz visible, pero mayor al del microondas), donde activan el estado vibracional de las moléculas y como resultado eleva la temperatura tanto de la superficie como en las capas internas de los granos en forma simultánea, lo que tiene efectos en el agua presente. De acuerdo con Pan y Atungulu (2011), esto se produce porque los enlaces $\mathrm{O}-\mathrm{H}$ del agua presente absorben la energía infrarroja y comienzan a rotar con la misma frecuencia que la radiación incidente. La transformación de la radiación infrarroja en energía rotacional causa la evaporación del agua desde el interior de los granos.

Al incrementar la temperatura del aire de secado y de la potencia de la radiación de los rayos infrarrojos en forma simultánea, se logra disminuir el tiempo de secado, hasta en un $77,7 \%$ y alcanzar 
humedades de equilibrio tan bajas como $0,05 \mathrm{~kg}$ $\mathrm{H}_{2} \mathrm{O} / \mathrm{kg}$ m.s. El menor tiempo de secado obtenido fue de $1,50 \mathrm{~h}$; utilizando $500 \mathrm{~W}$ de potencia con 60 ${ }^{\circ} \mathrm{C}$. La temperatura de aire de $60{ }^{\circ} \mathrm{C}$, genera un tiempo de secado menor (2,42 h), comparado con la temperatura de $50^{\circ} \mathrm{C}(3,166 \mathrm{~h})$ y $40^{\circ} \mathrm{C}(4,417 \mathrm{~h})$. La radiación infrarroja con potencia de $500 \mathrm{~W}$, genera un menor tiempo de secado $(2,167 \mathrm{~h})$, seguido por el de $250 \mathrm{~W}(2,667 \mathrm{~h})$ y finalmente $0 \mathrm{~W}$ $(5,167 \mathrm{~h})$. La combinación de temperatura de aire y potencia de radiación infrarroja que menor humedad de equilibrio alcanza en el de $0 \mathrm{~W}$ con 60 ${ }^{\circ} \mathrm{C}\left(0,05 \mathrm{~kg} \mathrm{H} \mathrm{H}_{2} \mathrm{O} / \mathrm{kg}\right.$ m.s. $)$.

\section{REFERENCIAS BIBLIOGRÁFICAS}

Borda, W., \& Gamarra, W. (2003). Diseño y Construcción de un equipo mejorado para el desaponificado de quinua (Chenopodium quinoa Willd.), Investigaciones Agroindustriales (Chenopodium quinoa Willd.) y Cañihua (Chenopodium pallidicaule Aellen). Puno, Perú.

Cerrón Mercado, F. (2013). Efectos de temperatura y tiempo en el desamargado y secado de quinua (Chenopodium quinoa Willd). Tesis para optar el título.Universidad Nacional del Centro del Perú. Huancayo, Perú.

Geankoplis, CH. (2002). Procesos de transporte y operaciones unitarias. Ed. CECSA. México.

Manco, José (1988). Interacción entre el Clima y Seis Variedades de Quinua en el Valle de Cajamarca. En Memorias del VI Congreso Internacional sobre Cultivos Andinos. Quito, Ecuador.

Miranda, M., Vega, A., López, J., Parada, G., Sanders, M., Aranda, M., Uribe, K., \& Di Scala, K. (2010). Impact of air-drying temperature on nutritional properties, total phenolic content and antioxidant capacity of quinoa seeds (Chenopodium quinoa Willd.). Industrial Crops and Products 32, 258-263.
Mujica, (1993). Reunión Técnica y Taller de Formulación del Proyecto Regional sobre Producción y Nutrición Humana en base a Cultivos Andinos. Arequipa, Perú.

Pan, Z., \& Atungulu, G. (2011). Infrared heating for food and agricultural processing. Boca Ratón. CRC Press. 284 p.

Perry, C. (1984). Chemical Engineers. Handbook, 5ta edición New York., McGraw- Hill.

Pereira, O., S. (2011). Elaboración de Leche de Quinua. Proyecto Previo a la Obtención del Título de Ingeniero Agroindustrial. Facultad de Ingeniería Química y Agroindustria Escuela Politécnica Nacional. Quito.

Quiroga Escalera \& Arteaga. (2010). Proyecto de Prefactibilidad para un proceso de beneficiado en seco de variedades amargas de quinua, basado en la aplicación de un lecho fluidizado de tipo surtidor. Universidad Privada Boliviana. Bolivia.

Romo, Sandra; Aura Rosero et al. (2006). Potencial nutricional de harinas de quínua (Chenopodium Quinoa Willd), variedad Partial en los Andes Colombianos Primera Parte. Facultad de Ciencias Agropecuarias. Vol. 4,(1).

Salas, Sonia. (2011). La Quinua. Post cosecha y Comercialización.

Soria, Marcial y Peñaloza. (1990). Lavado de Quinua. Procesos y Prototipos. Seminario Taller sobre Investigación de Producción de Quinua en Ecuador. Proyecto Procesamiento de Quinua en Ecuador. INIAP, UTA CIID. Quito.

Correo electrónico: jramos@uncp.edu.pe

Revisión de pares:

Recibido: 07/09/2019

Aceptado: 13/12/2019 